\title{
Co-activation and eEMG-feedback for Restoring Hand-Functions
}

\author{
Christian Klauer ${ }^{1}$, Emilia Ambrosini ${ }^{1}$, Simona Ferrante ${ }^{1}$, Alessandra Pedrocchi ${ }^{1}$ \\ ${ }^{1}$ Neuro Engineering And Medical Robotics Laboratory (NearLab), \\ Department of Electronics, Information and Bioengineering, Politecnico di Milano, Italy
}

\begin{abstract}
This contribution considers a neuroprosthesis for people who have (partially) paralyzed hand functions as a typical result of a stroke or spinal cord injury. Functional Electrical Stimulation (FES) is applied to three forearm-muscles to induce artificial muscle contractions causing wrist extension/flexion and grasping, respectively. The aim is to control and stabilize the wrist joint angle. Herein, a co-activation to increase the stiffness of the wrist joint is applied to yield a better tolerance to external influences. Doing so, a better motor precision is expected compared to other approaches. To maintain the level of co-activation also under the progression of muscle fatigue, the muscle activation is estimated and controlled using an underlying feedback of the electrical muscle responses as caused by motor unit recruitment. The control system was preliminary tested on two healthy subjects, and a wrist positioning error of $4.5^{\circ}$ on average was obtained demonstrating the feasibility of the co-activation based approach. ${ }^{1}$
\end{abstract}

\section{INTRODUCTION}

Neuroprosthetic systems are technical devices that electrically connect to the nervous system to obtain and influence sensory information or to induce motor functions. For the latter case, Functional Electrical Stimulation (FES) is a commonly used method that applies electrical pulses to the motor-nerves to trigger action potentials that generate muscle contractions. The application of the electricity is typically performed via surface electrodes [1].

To obtain or support functional movements, the stimulation intensity should be carefully adjusted by control algorithms. The realization of precise motor functions, however, is difficult to achieve as the musculoskeletal system is of high complexity and uncertainty. Therefore, most commercially available neuroprosthetic systems still apply FES in an open-loop manner without considering the generated movement [2]. In the restoration of hand functions, i.e., grasping and the stabilization/positioning of the wrist, fine motor control is beneficial. Therefore, in this contribution, we propose a new control scheme to improve the robustness against uncertainties and, hence, the motor precision in wrist movements.

Between 1989 and 1997, the Bionic glove was developed, which is a surrounding of the forearm combined with a stimulator to apply FES via surface electrodes [3]. The stimulation was turned on or off by special wrist movements that are caused by the volitional activity.

\footnotetext{
${ }^{1}$ Corresponding author Christian Klauer (chr.klauer@gmail.com). This work was supported by a postdoc fellowship of the German Academic Exchange Service (DAAD). We acknowledge Axelgaard Manufacturing Ltd for donating the stimulation electrodes. Further, we like to acknowledge Astrid Bergman for preparing the used inertial sensor set up.
}

As the influence of the extrinsic hand muscles on the grasping crosses the wrist joint, there is a tendency for the wrist to flex during grasping. As this shall be prevented because of physiological reasons, in 2000, a feedforward control using a neural network for compensation of unwanted wrist flexion was developed [4]. However, their investigations were only performed in simulation.

Few years later, feedback error learning control of the wrist angle using neural networks was considered [5]. Wellsufficient tracking of the wrist joint-angle was achieved after the neural network (NN) adapted itself based on the positioning error of a PID-controller. Though a pre-trained network might help, the convergence of learning is typically slow taking several minutes of continuous regular movements. As only sinusoidal references of fixed amplitude were applied in the learning it is, further, unsure how the $\mathrm{NN}$ performs in case of arbitrary reference trajectories. They found that PID-only control usually yields unsatisfying performance.

Recently, feedback-controlled grasping was considered in [6], [7]. Herein, a model predictive controller is presented to control the individual flexion angles of the fingers with satisfying performance. However, wrist angle control was not considered.

In [8] an approach for detecting the user-intention based on EMG and the wrist joint-angle is presented. The detector triggers FES to open the hand or to realize a grasp. Wrist angle stabilization was realized by constantly stimulating the wrist extensor during an active grasp. The authors report that FES shall be feedback controlled. A test in a stroke patient also indicates that the progression of muscle fatigue must be addressed as the performance decreased after a few successfully performed grasping tasks.

Despite all the investigations listed above, based on previous research [9], this contribution presents an approach using artificial co-activations to enhance the tolerance against uncertainties and external influences. This strategy mimics the natural generation of movements, wherein motor precision is achieved by a simultaneous contraction of multiple contrary acting muscles [10].

We exploit an increased joint stiffness to simplify the control of FES-induced movements. As the stiffness cannot be determined directly by an angle measurement, a feedback of the stimulation-evoked EMG is used to control the muscle's recruitment level in an underlying loop [11]. To control the wrist joint angle, feedforward and feedback control are combined in a sequential manner. Herein, open-loop control is applied to improve the tolerance to uncertainties 


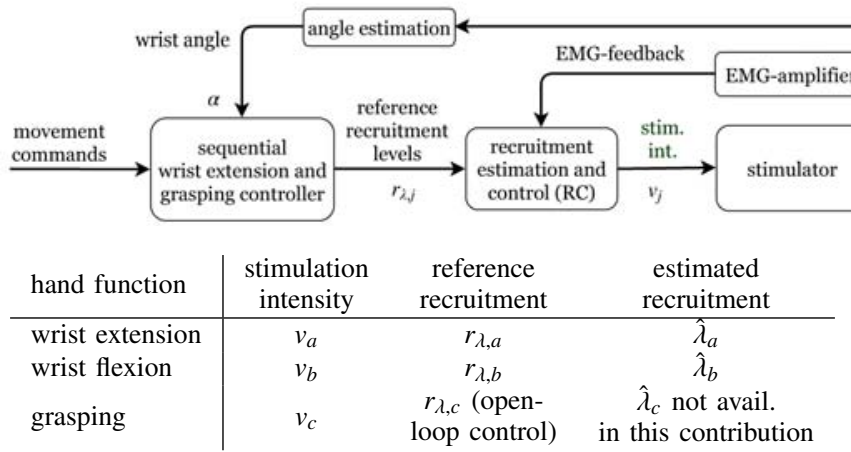

TABLE I

NotATion.

while approaching the target angle and - as the target is almost reached - feedback-control is activated to reduce the remaining positioning error. Grasping is controlled in an openloop manner, while stabilizing the wrist by feedback-control.

\section{System OVERVIEW}

An overview of the used set-up is shown in Fig. I. The wrist extension movements and grasping are solely generated by Functional Electrical Stimulation (FES). To realize hand-opening (wrist extension), closing (wrist flexion), and grasping, three muscles are stimulated.

This controller is implemented on a Linux-computer system that issues stimulation commands $v_{j} \in[0,1]$ for each muscle $j \in\{a, b, c\}$ to the stimulator (Rehastim, Hasomed $\mathrm{GmbH}$, Germany) and self-adhesive electrodes (ValuTrode $\AA$ CF4090 $(4 \times 9 \mathrm{~cm})$, Axelgaard Manufacturing Co., USA). Current amplitude and pulse width of the stimulation pulses are computed from the normalized ${ }^{2}$ pulse charge $v_{j}$ using the charge control method like in [11].

The wrist extension angle $\alpha[k]$ is measured at $100 \mathrm{~Hz}$ by means of two inertial sensors (MPU-9250, InvenSense Inc., USA). Further, Electromyography measurements (EMG) are used to determine the muscle-responses to FES using a multi-channel signal amplifier (Porti $32^{T M}$, TMS International, The Netherlands). The EMG-processing and recruitment control is implemented using OpenRTDynamics ${ }^{3}$ and the hand controller using Simulink employing a template for RTLinux code generation. To perform the calibration, to control experiments, and to visualize measured data, a browser-based GUI based on NodeJs is used.

A cascade control scheme is used: based on the measured EMG, the muscular recruitment level is estimated by digital filters for each muscle. Then, an underlying recruitment controller adjusts the stimulation intensities $v_{j}$ such that the desired recruitment levels $r_{\lambda, j}$ are realized, respectively. In an outer loop, the desired recruitments $r_{\lambda, j}$ are used to control the wrist joint angle $\alpha$ and grasping. A summary of the notation is given in Tab. I.

\footnotetext{
${ }^{2} v_{j}=1$ corresponds to the maximal tolerated stimulation intensity for the muscle $j$, respectively.

${ }^{3}$ OpenRTDynamics.github.io
}

Fig. 1 - The control scheme to realize hand functions: in an underlying loop, the muscle recruitment is controlled using a feedback of the electrical muscle responses to stimulation. In an outer loop, the wrist motion and grasping are controlled.

III. Methods

\section{A. Underlying recruitment control}

In previous research [11] we presented the recruitment control approach as an underlying controller for compensating muscle fatigue effects and reducing uncertainties in the generation of artificial muscle contractions. In FES, electrical pulses are periodically applied, whereby the period in-between two pulses is called inter-pulse interval (c.f. Fig. 2). Within this interval, the m-wave appears in the EMG-measurement whose amplitude depends on the number of motor units recruited by the previous stimulation pulse. Their typical duration is around $25 \mathrm{~ms}$. By taking the mean average value within a window that is optimized during a calibration procedure, the estimate $\hat{\lambda}$ is obtained ${ }^{4}$. Then $\hat{\lambda}_{i}$ is used as a feedback variable to adjust the stimulation intensity $v_{j}$ so that a desired recruitment level $r_{\lambda, j}$ is reached.

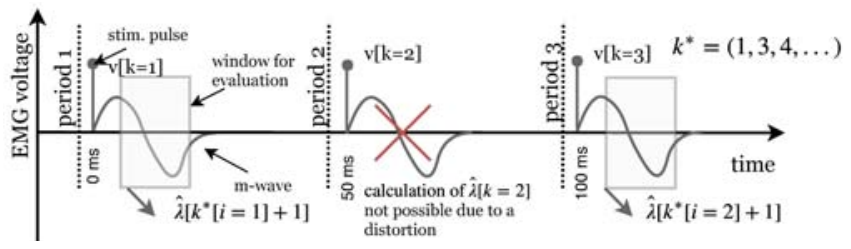

Fig. 2 - The m-waves caused by their belonging stimulation pulses.

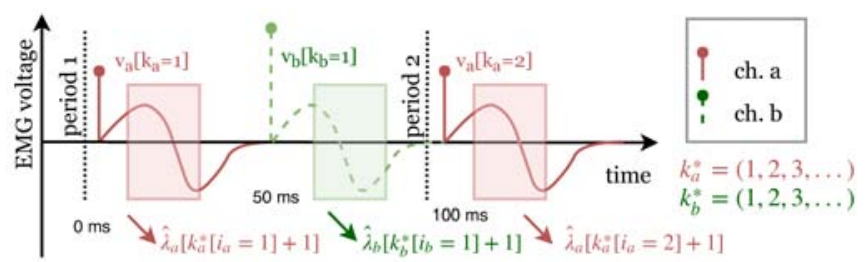

Fig. 3 - Time-multiplexed stimulation and measurement of the FES-evoked EMG: the measured EMG-signal is the superposition of the individual contributions of both muscle portions, respectively. As the stimulation alternates between both muscles, each inter-pulse interval only contains $\mathrm{m}$-waves solely caused by the belonging muscle. Therefore, the recruitment can be estimated.

Please note that the estimates for the recruitment level might not be available for each sampling instant, as the evaluation of the m-wave might fail, or when the feedbackcontrol shall be temporarily disabled (illustrated in Fig. 2). In any case, stimulation pulses must be applied, even when no estimates are available. Hence, in case of missing feedback, feedforward control will be applied. Two sampling indices are introduced: the sampling instants dedicated to the application of electrical pulses are denoted by $k$. The second set of sampling instants denoted by $i$ is introduced. It is assigned to the time instances of valid recruitment estimates. Missing

\footnotetext{
${ }^{4}$ The used optimization procedure, e.g., minimizes the noise variance of $\hat{\lambda}$ by varying the window's position and duration and is described in [11].
} 


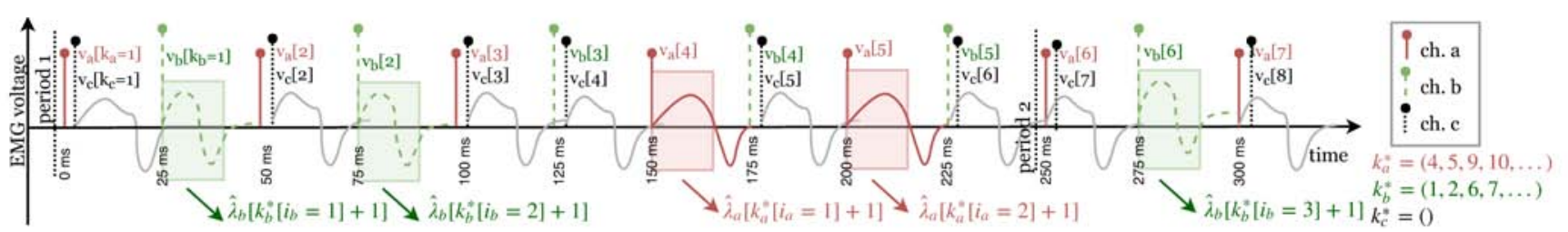

Fig. 4 - An almost regular pattern for applying stimulation pulses to three muscles. Within the inter-pulse intervals without overlap, estimates for the first and second muscle can be obtained (red and green windows). However, the estimation of the recruitment level for the third muscle is not possible.

updates are not counted by $i$. The sequence $k^{*}$ describes a mapping $k=k^{*}[i]$ between both types of sampling instants $i$ and $k$. The estimated recruitment $\hat{\lambda}\left[k^{*}[i]+1\right]$ depends on the previous stimulation pulse and its intensity $v\left[k^{*}[i]\right]$. As an estimate becomes available, the controller state is updated and the next intensity $v\left[k^{*}[i]+1\right]$ is calculated on that basis.

1) Two-channel stimulation: The application of contemporaneous multi-channel recruitment control to muscles close to each other ${ }^{5}$ is difficult as the m-waves evoked by the involved muscles typically overlap in the EMG measurement(s). Hence, the individual contributions cannot be distinguished. In the positioning of the wrist without grasping two muscles are concurrently stimulated. To treat overlap in two-channel stimulation, instead of applying pulses synchronously at the same time, the pulses are applied alternating among both stimulation channels as illustrated in Fig. 3. In effect, each muscle is stimulated at $20 \mathrm{~Hz}$. In turn, the respective m-waves do not overlap in the measured EMG. Further, it is possible to record the evoked EMG for multiple muscles using one pair of EMG electrodes if the muscles are sufficiently close to the EMG electrodes. The use of more than one electrode pair is still possible and might be used to improve the estimationaccuracy.

2) 3-channel stimulation and 2-channel EMG measurement: during grasping, three muscles must be concurrently stimulated. To allow the estimation of the recruitment, in this case, we propose the stimulation pattern given in Fig. 4. Herein, an important requirement is an (almost) regular stimulation at a frequency of at least $20 \mathrm{~Hz}$ in average, to prevent jitter in the muscle tone. In this pattern, muscles 1 and 2 are regularly stimulated ensuring a smooth muscle tone. However, the compromise is irregular and less frequent estimates for $\lambda_{1}$ and $\lambda_{2}$. Further, for $\lambda_{3}$ no estimates can be obtained. For the given application this is acceptable as grasping can be easily controlled in an open-loop manner.

The third muscle is stimulated slightly more often per period, however, still yielding a smooth tone. Please note that in this approach, the estimates of the muscular recruitment for muscles 1 and 2 are obtained non-regularly and less often: In the first half of one period, estimates for the second muscle $\hat{\lambda}_{b}$ are obtained, while during the second half estimates for the first muscle $\hat{\lambda}_{a}$ can be obtained.

3) Recruitment control for irregularly arriving estimates: The recruitment $\lambda$ caused by a previous pulse $v$ is described by a static non-linear recruitment function as illustrated in Fig. 5. Within a wide range for $v$, this function can be approximated

\footnotetext{
${ }^{5}$ Because all electrical potentials are spread within the surrounding tissue, the $\mathrm{m}$-waves produced by the individual muscles superpose. This causes the overlap in the measurement.
}

by the linear model

$$
\hat{\lambda}[k]=\Theta_{a} q^{-1} v[k]+\Theta_{b}+\eta[k], \quad \Theta_{r, a}>0 .
$$

Herein $\eta[k]$ describes gaussian noise, and $q^{-1}$ denotes the backward-shift operator $\left(s[k] q^{-1}=s[k-1]\right)$. The parameters $\Theta_{r, a}$ and $\Theta_{r, b}$ are adapted by a linear least squares approach matching recorded I/O data within the linear part region.

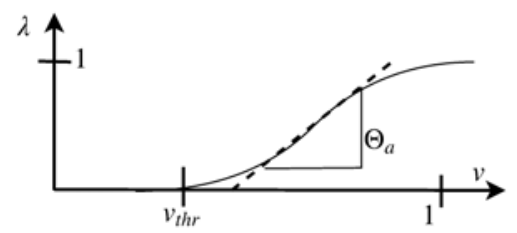

Fig. 5 - The muscular recruitment function and the used linear approximation. The stimulation intensity $v$ and recruitment $\lambda$ are normalized to their maximal values. Further, $v_{t h r}$ is the stimulation intensity yielding the onset of muscle contraction. It will be treated as a constant disturbance in control.

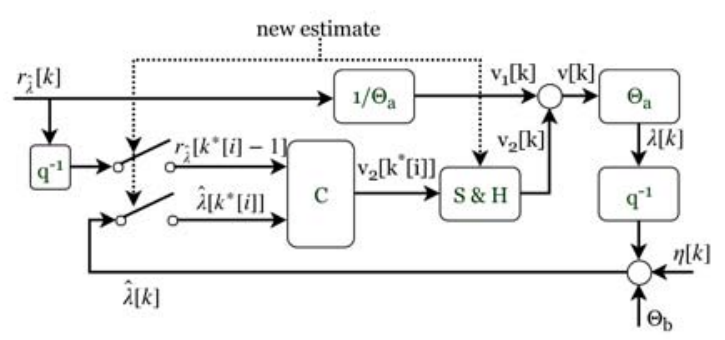

Fig. 6 - The structure of the recruitment controller that controls the recruitment level $\lambda$ of a muscle using its estimate $\hat{\lambda}$. This structure combines a feedforward path and the ability for triggered state updates as the estimates $\hat{\lambda}$ might be partially unavailable. If so, feedforward control is used.

To compensate for fatigue effects, uncertainties, and nonlinearities, a fast feedback loop is used to control $\hat{\lambda}$ by variating $v$. In comparison to [11], we improved this control scheme by adding a feedforward path and the ability to skip missing or faulty estimates as shown in Fig. 6. The feedforward path uses an inverse model $v_{1}[k]=r_{\lambda}[k] / \hat{\Theta}_{a}$. The feedback path uses the controller $C$ that is updated on the time instances $i$. Its actuation variable $v_{2}$ is held in case of missing updates. From the perspective of $C$, the augmented model, which includes the linear recruitment function, the feedforward path and the subsampling introduced by the mapping $k^{*}[i]$, is given by

$\hat{\lambda}\left[k^{*}[i]\right]=\eta\left[k^{*}[i]\right]+\Theta_{b}+\Theta_{a}\left(v_{2}\left[k^{*}[i]-1\right]+1 / \Theta_{a} r_{\lambda}\left[k^{*}[i]-1\right]\right)$.

Please note that because of the sample and hold implementation of the control variable, $v_{2}\left[k^{*}[i]-1\right]=v_{2}\left[k^{*}[i-1]\right]$ holds. Further, the reference applied one stimulation sampling instance before the arrival of the estimate $\bar{r}_{\lambda}\left[k^{*}[i]\right]:=$ $r_{\lambda}\left[k^{*}[i]-1\right]$ is introduced to simplify the notation. The control error that shall be compensated by $C$ is then given by

$$
\begin{aligned}
e\left[k^{*}[i]\right] & =\bar{r}_{\lambda}\left[k^{*}[i]\right]-\hat{\lambda}\left[k^{*}[i]\right] \\
& =-\eta\left[k^{*}[i]\right]-\Theta_{b}-\Theta_{a} v_{2}\left[k^{*}[i-1]\right] .
\end{aligned}
$$


This is achieved by choosing the integral controller

$$
v_{2}\left[k^{*}[i]\right]=\operatorname{sat}\left(v_{2}\left[k^{*}[i-1]\right]+\frac{k_{\lambda}}{\Theta_{a}}\left(\bar{r}_{\lambda}\left[k^{*}[i]\right]-\hat{\lambda}\left[k^{*}[i]\right]\right)\right) \text {. }
$$

The parameter $k_{\lambda}$ is determined by a pole-placement approach, such that the step-response (steps until $95 \%$ of the stationary value is reached) of the closed-loop behavior equals 4 sampling instants. The saturation function sat causes the state variable $v_{2}$ to remain within $[0,1]$.

\section{B. Hand function controller}

The control of wrist movements and grasping is performed using two separate controllers, respectively.

1) Wrist motion model: To describe the wrist joint angle $\alpha$, a linear, discrete-time model is used.

$$
\begin{array}{r}
\alpha_{T}[k]=\underbrace{m_{a}\left(\lambda_{a}[k]-\lambda_{a, c o}\right)}_{\alpha_{T, a}}+\underbrace{m_{b}\left(\lambda_{b}[k]-\lambda_{c, c o}\right)}_{\alpha_{T, b}}+\underbrace{m_{c} \lambda_{c}[k]}_{\alpha_{T, c}} \\
\alpha[k]=G_{m}\left(q^{-1}\right) \alpha_{T}[k], \quad G_{m}(q=1)=1 .
\end{array}
$$

The model is valid within a region close to the working point described by $\lambda_{a}=\lambda_{a, c o}$ and $\lambda_{b}=\lambda_{b, c o}$, within which the wrist is co-activated. Further, $\alpha_{T, a}$, and $\alpha_{T, b}$ describe the contributions of the wrist flexor- and extensor muscles weighted by the factors $m_{a}>0, m_{b}<0$, respectively. Since the stimulation of the muscle causing a grasp typically causes also the wrist to flex, $\alpha_{T, c}$ and $m_{c}<0$ are introduced. The dynamics of the wrist motion are described by a second-order transfer function that has unity static gain $\left(G_{m}(1)=1\right)$ :

$$
G_{m}\left(q^{-1}\right)=q^{-2} \frac{1-a_{1}-a_{2}}{1+a_{1} q^{-1}+a_{2} q^{-2}} .
$$

Hence, $\alpha_{T}$ describes the joint angle in which the system $G_{m}$ is stationary. The parameters $a_{1}$ and $b_{2}$ are determined by a system identification approach. It is assumed that the dynamics of the underlying recruitment controllers are significantly faster than $G_{m}$. Therefore, $r_{\lambda_{a}}=\lambda_{a}$ and $r_{\lambda_{b}}=\lambda_{b}$ are assumed.

2) Static inversion: The 2-DoF actuation using $r_{\lambda_{a}}$ and $r_{\lambda_{b}}$ allows manipulating the angle and the level of co-activation ${ }^{6}$ independently. Therefore, we propose the mapping shown in Fig. 7 that introduces an augmented actuation variable $u \in\left[u_{\min }, u_{\text {max }}\right]$ to influence the joint angle $\alpha$ while modulating the co-activation. Herein, the parameters $\lambda_{a, c o}$ and $\lambda_{b, c o}$ define the co-activation level of the working point. By setting $f_{a}=$ $f_{b}=0.3$, the level of co-activation reduces to $30 \%$ of its original value for $(u=0)$ as the control variable approaches the constraints $u_{\text {min }}$ and $u_{\text {max }}$, respectively.

The parameters $c_{a}, c_{b}$ shall be chosen such that the static linear part of the model described by Eq. (2) is inverted in case of inactive grasping $\left(\lambda_{c}=0\right)$ so that $u=\alpha_{T}$ is obtained. For $u>0$ (Case (A)), the equations to calculate the desired recruitment levels $r_{\lambda, a}$ and $r_{\lambda, b}$ are then given by:

$$
\begin{aligned}
& r_{\lambda, a}=\lambda_{a}=\lambda_{a, c o}+c_{a} u \\
& r_{\lambda_{b}}=\lambda_{b}=\lambda_{b, c o}-g_{b} u
\end{aligned}
$$

${ }^{6}$ Def.: $\alpha_{T, c o}:=\min \left(m_{a} \lambda_{a}, m_{b} \lambda_{b}\right)$.

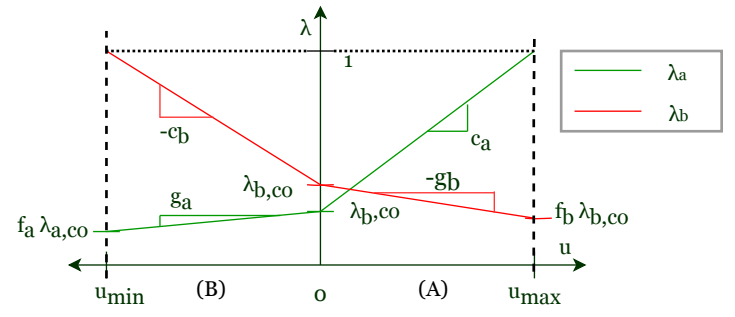

Fig. 7 - The mapping strategy to calculate the muscle recruitments $\lambda_{a}$ and $\lambda_{b}$ maintaining the co-activation based on an augmented control variable $u$.

The limitation to a maximal recruitment $\lambda_{a} \leq 1$ also implies a limit for $u$ :

$$
u \leq u_{\max }=\left(1-\lambda_{a, c o}\right) / c_{a} .
$$

The parameter $g_{b}$ is then given by

$$
g_{b}=\lambda_{b, c o}\left(1-f_{b}\right) / u_{\max }, 0<f_{b} \leq 1 .
$$

In case $(\mathrm{B})(u<0)$, similar equations are obtained:

$$
\begin{aligned}
r_{\lambda, a} & =\lambda_{a}=\lambda_{a, c o}+g_{a} u \\
r_{\lambda_{b}} & =\lambda_{b}=\lambda_{b, c o}-c_{b} u \\
u & \geq u_{\min }=\left(\lambda_{b, c o}-1\right) / c_{b} \\
g_{a} & =\lambda_{a, c o}\left(f_{a}-1\right) / u_{\min }, 0<f_{a} \leq 1
\end{aligned}
$$

By applying the proposed control law (Eqs. (5), (6)) to Eq. (2) in case (A)

$$
T_{\alpha}=m_{a} c_{a} u+m_{b} g_{b} u
$$

results. Similar, in case (B)

$$
T_{\alpha}=m_{a} g_{a} u+m_{b} c_{b} u
$$

is obtained. To achieve exact inversion in the stationary case $\left(T_{\alpha}=u\right)$, the conditions

$$
1=m_{a} c_{a}+m_{b} g_{b}, \quad 1=m_{a} g_{a} u+m_{b} c_{b} u
$$

must be fulfilled. In a calibration procedure, the controller parameters $c_{a}$ and $c_{b}$ are directly obtained experimentally. Then, the model parameters $m_{a}$ and $m_{b}$ can be calculated by

$$
m_{a}=\frac{g_{b}-c_{b}}{g_{a} g_{b}-c_{a} c_{b}}, \quad m_{b}=\frac{g_{a}-c_{a}}{g_{a} g_{b}-c_{a} c_{b}} .
$$

3) Wrist motion dynamics compensation: An inversion of the wrist motion dynamics $G_{m}$ (c.f. Eq. (4)) is applied as shown in Fig. 8. Herein, the static inverse (c.f. Fig. 7) is applied to the model (2), (3) yielding $T_{\alpha}=u$. To perform an inversion of $G_{m}$ an approximate, causal inverse

$$
G_{m}^{*}\left(q^{-1}\right)=\frac{q^{-2}}{G_{m}\left(q^{-1}\right)}=\frac{1+a_{1} q^{-1}+a_{2} q^{-2}}{1-a_{1}-a_{2}}
$$

is used. Herein, two poles at zero (introducing a delay of two sampling instants) are used so that the $G_{m}^{*}$ is causal. Please note that this approach exactly cancels the poles of the dynamics $G_{m}$. This typically yields a dead-beat behavior and, hence, tends to increase the amplitude of the actuation. Therefore, in a next step, the component of the control signal 


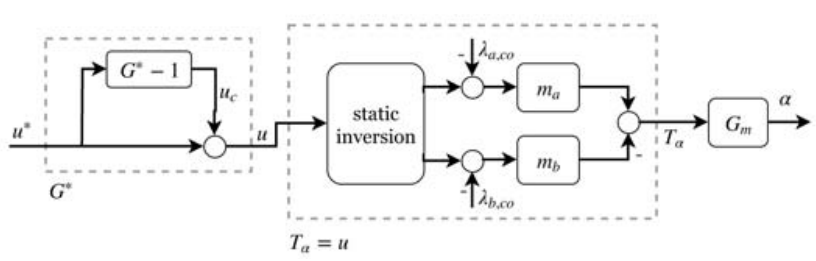

Fig. 8 - The inversion of the wrist movement dynamics $G_{m}$.

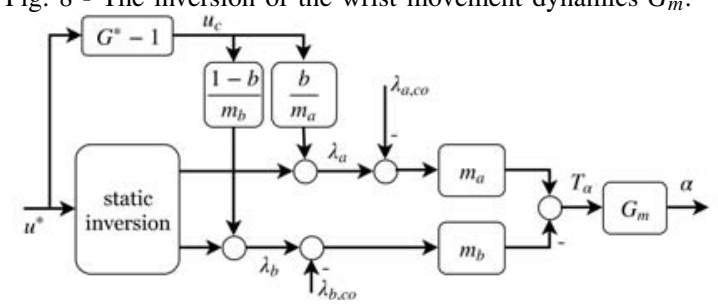

Fig. 9 - Equivalent re-arrangement of Fig. 8: the inverse dynamics is directly applied and distributed among the muscle recruitment levels $\lambda_{a}$ and $\lambda_{b}$.

for realizing the inversion is separated ${ }^{7}$ and is distributed across both muscles as shown in Fig. 9. Herein, the factor $b \in$ $[0,1]$ specifies a balance-factor for the distribution among both muscles. In this contribution, we chose an equal distribution $(b=0.5)$. Please note that both realizations are equivalent with regard to the inverted system behavior in between the newly introduced actuation variable $u^{*}$ and $\alpha$.

4) Wrist postion control: To realize a positioning of the wrist angle $\alpha$ according to the reference $r_{\alpha}$, the switching control scheme shown in Fig. 10 is used. The following sequence of controllers is used to approach the target position:

1) By applying $d u_{o l}=8^{\circ} / \mathrm{s}$ to the integrator (causing $u^{*}$ to linearly increase), the target is linearly approached by open-loop control. Once the measured angle enters an error band $-15^{\circ}<e_{\alpha}<-15^{\circ}$, step 2) is activated.

2) Integral feedback-control is applied until the measured angle enters the error band $-5^{\circ}<e_{\alpha}<-5^{\circ}$ and the wrist movement stopped ${ }^{8}$. Then step 3 ) is activated. In any case, after 8s step 3) is activated too (timeout).

3) It is assumed that the desired wrist postured is reached and, hence, $u^{*}$ is kept constant. Changes in the wrist posture are prevented due to the stiffness introduced by the co-activation.

\footnotetext{
${ }^{7}$ Without the dynamics' compensation, $u=u^{*}$ would be applied. Now, this becomes $u=G^{*} u^{*}=\left(1+\left(G^{*}-1\right)\right) u^{*}=u^{*}+u_{c}$, wherein $u_{c}=G^{*}-1$ is an additional portion present in the actuation signal that is responsible for the compensation of the dynamics. The influence of $u_{c}$ shall be spread across both muscles while the effect of the compensation remains.

${ }^{8}$ When $\alpha$ does not change by more than $1^{\circ}$ within $0.5 \mathrm{~s}$, the movement is considered to have stopped.
}

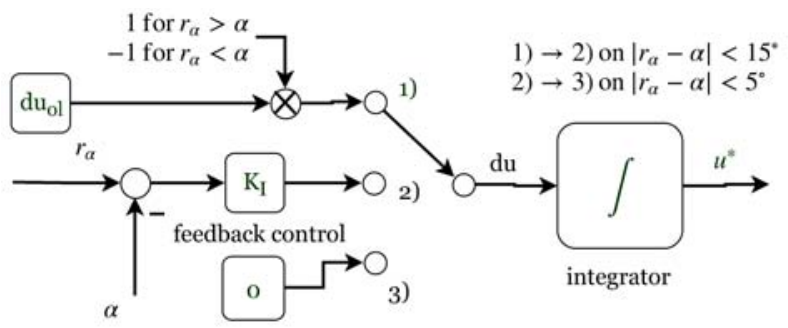

Fig. 10 - The used controller for positioning the wrist: a sequential strategy involving 1) open-loop, and ongoing 2) feedback-control is used to reach the given target angle $r_{\alpha}$. In state 3 ) the wrist stiffness prevents changes of the wrist posture.
The integral controller gain $K_{I}=0.4$ yields a step response of $150 \mathrm{~ms}$ when applied to the nominal model.

5) Grasping: To perform a grasp, $r_{\lambda, c}$ is linearly increased up to $\lambda_{g r}$ within $3.5 \mathrm{~s}$. Similarly, to release the grasp, $r_{\lambda, c}$ is linearly decreased till 0 . To compensate the influence of the grasping on the wrist angle, as assumed in the model 2 , the integral position controller is activated during the transitions between grasping and releasing, as well as for $2 \mathrm{~s}$ after the transitions are completed. During grasping, the stimulation pattern described in III-A.2 is applied. The feedback part of the recruitment controller is deactivated.

\section{Calibration}

To obtain the optimal window parameters for calculating the recruitment estimates $\hat{\lambda}_{j}$, and the parameters $\Theta_{a, j}, \Theta_{b, j}$ of the linear recruitment model, the calibration routine described in [11] is applied for each muscle $j$.

For the hand controller, the co-activation level described by the parameters $\lambda_{a, c o}$ and $\lambda_{b, c o}$ of the wrist joint is determined. Therefore a test is performed in which, $r_{\lambda, b}$ remains zero, while $r_{\lambda, a}$ is linearly increased till the wrist angle reaches $20^{\circ}$ yielding $\lambda_{a, c o}$. Then, while applying $r_{\lambda, a}=\lambda_{a, c o}, r_{\lambda, b}$ is linearly increased till the wrist joint reduces to $0^{\circ}$ yielding $\lambda_{b, c o}$. This causes a slight co-activation in the wrist joint.

In a next test, the gain factors $c_{a}$ and $c_{b}$ of the static mapping strategy (Fig. 7) are determined. Therefore, openloop control is used by directly applying a step-wise changing signal to the augmented actuation variable $u$ (dynamic compensation not active). The aim is to obtain $u \approx \alpha$ in the stationary case. The plateaus of the steps are chosen to be $-20^{\circ}$ and $20^{\circ}$ degrees, respectively. During this procedure that starts with $c_{a}=c_{b}=0$, these factors are increased by trial-and-error approach until the measured angle $\alpha$ closely matches the reference $u$ in the respective steady-states after each step. To determine $G_{m}$, I/O-data obtained for the step responses is used to identify the parameters $a_{1}$ and $a_{2}$ by minimizing the quadratic error of the responses.

The recruitment level required to induce a gasp $\lambda_{g r}$ is determined by manually increasing $r_{\lambda, c}$ while the wrist joint is co-activated. As the fingers are sufficiently closed, $\lambda_{g r}$ is obtained.

\section{Results}

The proposed controller was tested in two healthy subjects. Fig. 11 shows time series results for a test sequence to mimic approaching, grasping and releasing of an object. Data of the wrist extension angle, the muscle recruitment level, and the stimulation intensity are given for one trial, respectively.

Because of likely disturbed EMG-measurements during the grasping phases, we decided to deactivate the EMG feedback (c.f. Sec. III-A.3) for this contribution as we cannot guarantee the proper estimation of $\lambda$ in this case. However, we recorded the data for monitoring and evaluation purposes.

The recruitment controllers are able to track the given references, when feedback control is active. In both cases a) and b), the target angles are reached within $9.2 \mathrm{~s}$ and $9.1 \mathrm{~s}$ in average, respectively. The remaining angle error was $4.46^{\circ}$ 
and $4.5^{\circ}$ in average for a) and b), respectively. We observe a maximal drop of the wrist angle of $13.4^{\circ}$ and $29.7^{\circ}$ as grasping is performed.
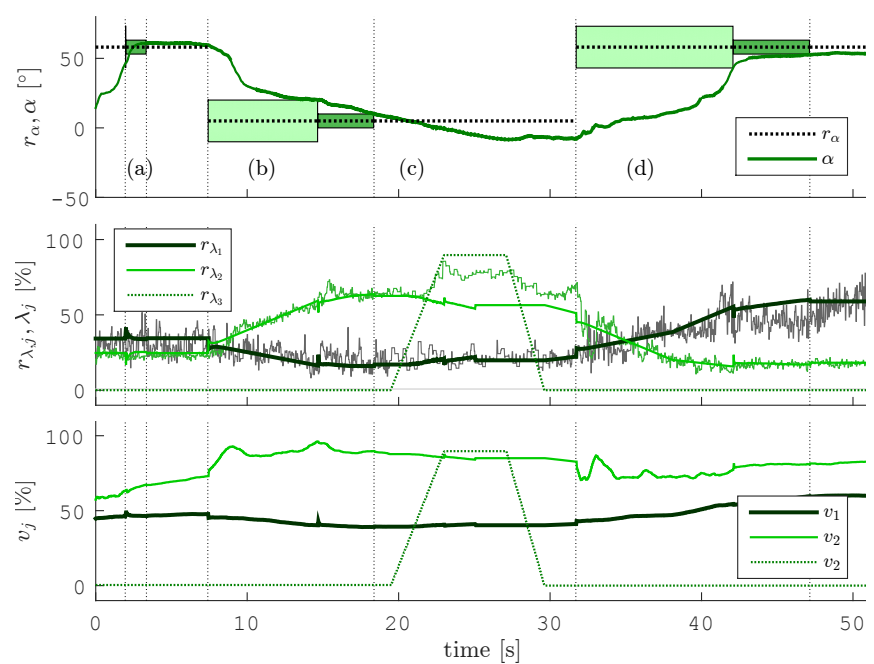

a)
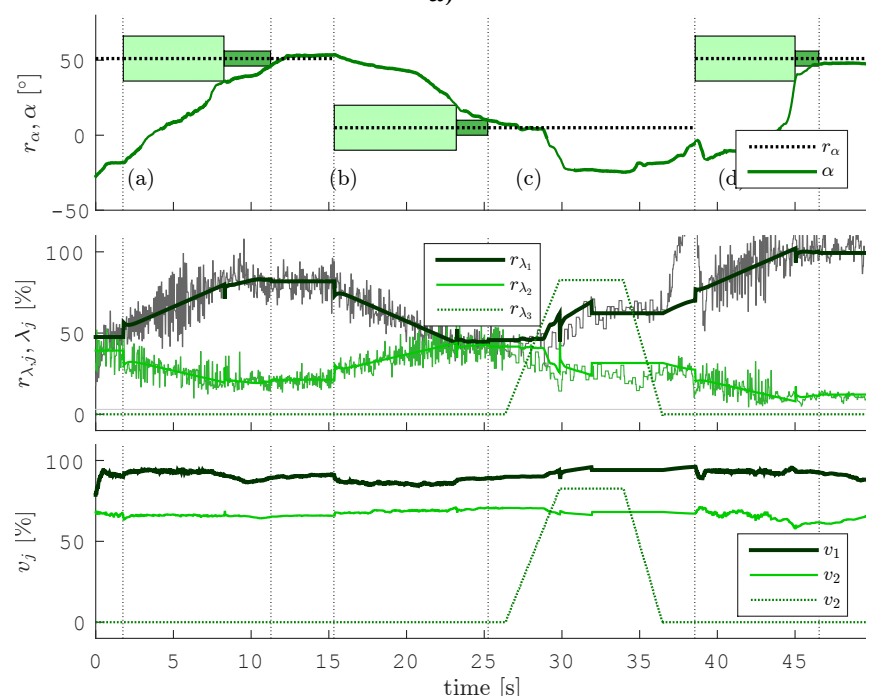

b)

Fig. 11 - Exemplary time series data for an approaching (a)/(b) - grasping/releasing (c) - hand opening (d) sequence in two healthy subjects (a) age 34 years, male, b) age 24 years, female). Shown are the wirst angle $\alpha$ and its target $r_{\alpha}$. The green areas indicate the tolerance bands introduced in Sec. III-B.4. The thin, solid lines are the estimated recruitment levels $\hat{\lambda}_{j}$.

\section{Conclusions and Outlook}

For the restoration of grasping in daily life, the obtained accuracy of the co-activation based wrist positioning of less than five degrees seems feasible. However, some improvements have to be carried out: The positioning time, for example, shall be further improved by optimizing the factor $d u_{o l}$. Herein, the limits of a smooth control of movements should be investigated. Moreover, the wrist stabilization during grasping should be further improved. This might be achieved by compensating the influence of grasping on the wrist angle using additional feed-forward control. In order to avoid another step in the calibration to obtain the parameter $m_{c}$, a run-to-run control might be applied to iteratively adapt a feedforward control portion.
It was observed that the amplitude of the m-waves was typically low in comparison to other artifacts also present in the EMG-signal. ${ }^{9}$ This might cause a low signal-to-noise ratio in the estimate of the recruitment level and, hence, yield an insufficient control performance. We observed that with the stimulation of three muscles, more distortion is typically present in the EMG signal. Hence, for this contribution, we decided to disable the EMG-feedback during grasping. In order to activate the latter, more effort must be spend for signal processing of the raw EMG signals.

In addition to the stimulation patterns illustrated in Figs. 4 and 3 , other stimulation patterns might be considered in order to obtain recruitment estimates for all muscles. So far, fixed durations for the inter-pulse intervals are used. To enhance the smoothness of the muscle tone, these durations might be optimized (i.e. shortened) to achieve a higher effective stimulation frequency. We tested a pattern with which an EMG-measurement from all muscles is possible (not presented). Herein, further timing optimizations are required to remove jitter in the muscle tone.

\section{REFERENCES}

[1] D. N. Rushton, "Functional electrical stimulation," Physiological Measurement, vol. 18, pp. 241-275, Nov. 1997.

[2] C. Lynch and M. Popovic, "Closed-loop control for fes: Past work and future directions," in 10th Annual Conference of the International FES Society, 2005.

[3] A. Prochazka, M. Gauthier, M. Wieler, and Z. Kenwell, "The bionic glove: An electrical stimulator garment that provides controlled grasp and hand opening in quadriplegia," Archives of Physical Medicine and Rehabilitation, vol. 78, no. 6, pp. 608 - 614, 1997.

[4] M. M. Adamczyk and P. E. Crago, "Simulated feedforward neural network coordination of hand grasp and wrist angle in a neuroprosthesis," IEEE Transactions on Rehabilitation Engineering, vol. 8, no. 3, pp. 297-304, Sept 2000.

[5] K. Kurosawa, R. Futami, T. Watanabe, and N. Hoshimiya, "Joint angle control by fes using a feedback error learning controller," IEEE Transactions on Neural Systems and Rehabilitation Engineering, vol. 13, no. 3, pp. 359-371, Sept 2005.

[6] A. J. Westerveld, A. C. Schouten, P. H. Veltink, and H. van der Kooij, "Passive reach and grasp with functional electrical stimulation and robotic arm support," in 2014 36th Annual International Conference of the IEEE Engineering in Medicine and Biology Society, Aug 2014, pp. 3085-3089.

[7] A. J. Westerveld, A. Kuck, A. C. Schouten, P. H. Veltink, and H. van der Kooij, "Grasp and release with surface functional electrical stimulation using a model predictive control approach," in 2012 Annual International Conference of the IEEE Engineering in Medicine and Biology Society, Aug 2012, pp. 333-336.

[8] C. Salchow, A. Dorn, M. Valtin, and T. Schauer, "Intention recognition for fes in a grasp-and-release task using volitional emg and inertial sensors," Current Directions in Biomedical Engineering, vol. 3, no. 2, p. 161-165, 2017.

[9] M. Ruppel, C. Klauer, and T. Schauer, "Towards a high motorprecision neuroprosthesis by recruitment-controlled antagonistic muscle co-activation strategies," in Abstract in Proc. of the 6th European Conference on Technically Assisted Rehabilitation - TAR 2017, Berlin, Germany, 2017.

[10] E. Burdet, R. Osu, D. W. Franklin, T. E. Milner, and M. Kawato, "The central nervous system stabilizes unstable dynamics by learning optimal impedance," Nature, vol. 414, no. 6862, pp. 446-449, 2001.

[11] C. Klauer, S. Ferrante, E. Ambrosini, U. Shiri, F. Dähne, I. Schmehl, A. Pedrocchi, and T. Schauer, "A patient-controlled functional electrical stimulation system for arm weight relief," Medical Engineering $\mathcal{E}$ Physics, vol. 38, no. 11, pp. 1232-1243, 2016.

${ }^{9}$ In contrast and in comparison to previous research, the m-waves typically obtained from the shoulder deltoid, are less affected by artifacts, because of their higher amplitude. 